\title{
Expression profile of plasma microRNAs in premature infants with respiratory distress syndrome
}

\author{
QING KAN $^{1 *}$, SUFANG DING $^{2^{*}}$, YANG YANG $^{1}$ and XIAOYU ZHOU ${ }^{1}$ \\ ${ }^{1}$ Department of Neonatology, Nanjing Children's Hospital Affiliated to Nanjing Medical University, Nanjing, Jiangsu 210008; \\ ${ }^{2}$ Department of Neonatology, Huaian Maternity and Child Health Care Hospital, Huaian, Jiangsu 223002, P.R. China
}

Received May 21, 2014; Accepted March 20, 2015

DOI: $10.3892 / \mathrm{mmr} .2015 .3699$

\begin{abstract}
As well-known regulators of gene expression, microRNAs (miRNAs) are important not only in cell proliferation and differentiation, but also in tumorigenesis and organ development. It has been estimated that miRNAs may be responsible for regulating the expression of almost one third of the human genome. Simultaneously, with advances in neonatal care in the clinic, an increased number of premature infants are being saved and, thus, respiratory distress syndrome (RDS) has become more common. However, previous non-miRNA studies have suggested their connection with RDS. In the present study, a miRNA microarray, including $>1,891$ capture probes was used to compared the expression profiles of plasma miRNAs between RDS and control groups. miRNAs, which were observed to have consistent fold-changes (fold-change $\geq 1.3$ ) between the two groups were selected and validated using reverse transcription-quantitative polymerase chain reaction. As a result, 171 differentially expressed miRNAs were identified, including two upregulated and seven downregulated miRNAs. Of these miRNAs, four were selected as having higher fold-changes between the two groups. This is the first time, to the best ouf our knowledge, that these nine miRNAs have been reported in RDS. It was hypothesized that these novel miRNAs may be important in RDS, and may provide meaningful biomarkers for the diagnosis of RDS.
\end{abstract}

\section{Introduction}

Respiratory distress syndrome (RDS) is a severe type of respiratory disease, characterized by a lack of pulmonary surfactant (PS). As an increasingly common syndrome in preterm infants, without effective treatment, progressive dyspnea, expiratory

Correspondence to: Ms. Xiaoyu Zhou, Department of Neonatology, Nanjing Children's Hospital Affiliated to Nanjing Medical University, 72 Guangzhou Road, Nanjing, Jiangsu 210008, P.R. China

E-mail: xyzhou161@163.com

${ }^{*}$ Contributed equally

Key words: microRNA, premature, respiratory distress syndrome groaning and respiratory failure can appear just a few hours following birth (1). Treatment of this disease has remained difficult and although the assistance of PS and mechanical ventilation has resulted in a marked reduction in mortality rates, the incidence of RDS does not follow a downward trend due to increased survival rates of low birth weight premature infants (2).

The development of respiratory system begins at a gestational age (GA) of 3-4 weeks, which originated from the endodermal germ layer of the embryo. In humans, this is divided into six stages (3): Embryonic stage (GA of 3-7 weeks), pseudoglandular stage (GA of 7-16 weeks), canalicular stage (GA of 16-24 weeks), saccular stage (GA of 24 weeks to birth), alveolar stage and microvascular maturity stage. Among these, the earlier four stages occur in the uterus and are more likely to be affected by the complex extrauterine environment (3). The entire process of lung development is highly regulated, with the involvement of signal transduction molecules secreted from lung epithelial cells and interstitial cells, including WNT, BMP-4, TGF- $\beta$, SHH, FGF (3). In several situations, these signal molecules are further regulated by certain types of RNAs to guarantee developmental coordination in time and space. Micro (mi)RNAs are one of the small RNAs involved in lung development and diseases (4-8).

miRNAs are a type of endogenous, non-coding, single-stranded RNA molecule. It modulates the activity of specific mRNA targets at the post-transcription level, and are thus important in a wide range of physiological and pathological processes (9), including cell differentiation, cell proliferation, cell cycle regulation and organ development. miRNAs are highly conserved between different mammals and target almost $30 \%$ of the protein-coding genes in humans, including transcription factors, signaling proteins, receptors and metabolic enzymes (10-13). miRNA expression levels vary markedly among tissues and it has been suggested that dysregulation of miRNAs can contribute to pathogenic diseases (14).

In vivo, there are several resources of miRNAs, including tissue miRNAs and circulating miRNAs. As early as 1948, Mandel and Paris had demonstrated that RNAs are common in serum and plasma (15). Notably, miRNAs are also present in large quantities in the blood. It exhibits high stability to avoid blood damage from RNases following PH change or repeated freezing and thawing $(16,17)$. The source of miRNAs 
remain to be fully elucidated, however they may be from apoptotic or necrotic cells (18). Although there have been no previous reports regarding plasma miRNAs in RDS, those of other diseases can be of assistance. Ai et al demonstrated that, in the plasma of patients with myocardial infarction, the expression level of miRNA-1 was at the same high level as in the myocardium (19). Taylor et al (12) also reported higher specific cancer-associated miRNAs in the peripheral blood of patients with ovarian cancer, from exosomes, compared with patients with benign diseases. Therefore, theoretically, plasma miRNAs may be a suitable potential biomarker in the diagnosis of clinical diseases. With the assistance of microarrays and subsequent reverse transcription-quantitative polymerase chain reaction (RT-qPCR), the present study aimed to identify significantly expressed miRNAs in the plasma of infants with RDS, compared with normal premature infants.

\section{Materials and methods}

Ethical statement. The present study was approved by the Medical Ethics Committee of Nanjing Medical University Affiliated Nanjing Children's Hospital (Nanjing, China; permit number 201002008). Written informed consent was obtained from the guardians on behalf of the infants enrolled in the present study. All clinical investigations were performed according to the principles expressed in the Declaration of Helsinki (20). The guardians had the right to withdraw from the study at any time. Initially, 22 infants with RDS were recruited, however, the guardians of two of the infants withdrew from the investigation..

Patients. A total of 20 infants with RDS and 29 infants without RDS (controls) at a GA of 28-34 weeks. were recruited between October 2010 and May 2011 from the Neonatal Intensive Care Unit of Nanjing Children's Hospital. Written, informed consent was obtained from the family of the patient.

Inclusion criteria. In the RDS group the diagnostic criteria for RDS were as follows: GA between 28 and 34 weeks, classic symptoms of progressive dyspnea and corresponding X-ray signs, and required assistant ventilation via continuous positive airway pressure or mechanical ventilation. The control group comprised normal premature infants without breathing difficulty breathing or signs of RDS on X-ray.

Exclusion criterion. The criteria for exclusion in the present study included: The application of cortical hormone prior to or following birth, severe deformity or chromosomal abnormalities, a diagnosis of congenital adrenal hyperplasia, severe perinatal asphyxia or repeated hypoglycemia.

Sample collection. Fasting venous blood samples were collected from the infants in the morning between 7.00 and $9.00 \mathrm{am}$ ) on the 1st, 4th and 10th days following birth. Subsequently, the blood samples were transferred into EDTA anticoagulant tubes and centrifuged (CS-15R; Beckman Coulter, Inc., Fullerton, CA, USA) for 5 mins at $1,006.2 \mathrm{x} \mathrm{g}$. The plasma was subsequently maintained in a refrigerator at $4^{\circ} \mathrm{C}$ for further experiments.
RNA extraction. The total RNA was isolated from the plasma using TRIzol (Invitrogen Life Technologies, Carslbad, CA, USA) and an miRNeasy Mini kit (Qiagen, Hilden, Germany), according to manufacturer's instructions. The RNA quality and quantity was measured using a nanodrop spectrophotometer (ND-1000; Thermo Fisher Scientific, Wilmington, DE, USA) and the RNA integrity was determined using $1 \%$ agarose gel electrophoresis.

miRNA microarray. Following RNA isolation, an miRCURY ${ }^{\mathrm{TM}}$ Hy $3^{\mathrm{Tм}} / \mathrm{Hy}^{\mathrm{Tм}}$ Power Labeling kit (Exiqon, Inc., Vedbaek, Denmark) was used, according to the manufacturer's instructions for miRNA labeling. A total of three slides were used between the RDS group and control group. For each slide, $1 \mu \mathrm{g}$ of each sample was $3^{\prime}$-end-labeled with $\mathrm{Hy}^{\mathrm{TM}}$ fluorescent label, using T4 RNA ligase in the following procedure: The RNA, in $2.0 \mu \mathrm{l}$ water, was combined with $1.0 \mu \mathrm{l}$ CIP buffer and CIP (Exiqon, Inc.). The mixture was incubated for $30 \mathrm{~min}$ at $37^{\circ} \mathrm{C}$, and was terminated by incubation for $5 \mathrm{~min}$ at $95^{\circ} \mathrm{C}$. Subsequently, $3.0 \mu 1$ labeling buffer, $1.5 \mu$ l fluorescent label $\left(\mathrm{Hy} 3^{\mathrm{TM}}\right), 2.0 \mu 1$ dimethyl sulfoxide (DMSO) and $2.0 \mu \mathrm{l}$ labeling enzyme were added to the mixture. The labeling reaction was incubated for $1 \mathrm{~h}$ at $16^{\circ} \mathrm{C}$, and terminated by incubation for $15 \mathrm{~min}$ at $65^{\circ} \mathrm{C}$.

Following termination of the labeling procedure, the $\mathrm{Hy} 3^{\mathrm{TM}}$-labeled samples were hybridized on the miRCURY ${ }^{\mathrm{TM}}$ LNA Array (v.16.0; Exiqon, Inc.), according to manufacturer's instructions. The $25 \mu \mathrm{l}$ mixture from the $\mathrm{Hy} 3^{\mathrm{TM}}$-labeled samples were added to $25 \mu 1$ hybridization buffer and were denatured for $2 \mathrm{~min}$ at $95^{\circ} \mathrm{C}$, incubated on ice for $2 \mathrm{~min}$ and then hybridized to the microarray for $16-20 \mathrm{~h}$ at $56^{\circ} \mathrm{C}$ in a 12 -Bay Hybridization system (Nimblegen Systems, Inc., Madison, WI, USA), which provides an active mixing action and constant incubation temperature to improve hybridization uniformity and enhance signal. Following hybridization, the slides were washed several times using a wash buffer kit (Exiqon, Inc.), and finally dried by centrifugation for $5 \mathrm{~min}$ at $134.1 \mathrm{x} \mathrm{g}$. The slides were then scanned using an Axon GenePix 4000B Microarray Scanner (Axon Instruments, Foster City, CA, USA).

The scanned images were imported into GenePix Pro 6.0 software (Axon Instruments) for grid alignment and data extraction. The replicated miRNAs were averaged and miRNAs with intensities $>50$ in all the samples were selected for calculation of the normalization factor. The data were normalized using the median normalization. Following normalization, differentially expressed miRNAs were identified through Volcano Plot filtering (fold-change $\geq 1.3 ; \mathrm{P} \leq 0.05$ ). In addition, hierarchical clustering was performed using MEV software (v4.6; TIGR, Boston, MA, USA).

$R T$ - $q P C R$. Following isolation of the RNA from the plasma using TRIzol reagent, single-strand cDNA was synthesized as follows: The RT mixture contained $1 \mu \mathrm{g}$ total RNA, $0.3 \mu \mathrm{l}$ rno-miRNA reverse primer $(1 \mu \mathrm{M}), 0.1 \mu \mathrm{l}$ MMLV Revertase (200 U/ $\mu 1$; Epicentre, Madison, WI, USA), $2 \mu \mathrm{l}$ 10X RT Buffer, $2 \mu \mathrm{l}$ dNTP mix (2.5 mM each; HyTest, Ltd., Turku, Finalnd) and $0.3 \mu \mathrm{l}$ ribonuclease inhibitor (40 U/ $\mu 1$; Epicentre), in a $20 \mu 1$ total volume. The reaction was performed at $16^{\circ} \mathrm{C}$ for $30 \mathrm{~min}$ and at $42^{\circ} \mathrm{C}$ for $40 \mathrm{~min}$, 
Table I. Primers used for reverse transcription-quantitative polymerase chain reaction.

microRNA
Primer $\left(5^{\prime}-3^{\prime}\right)$

\section{U6}

hsa-miR-301a

hsa-miR-513a-3p

hsa-miR-3679-3p

hsa-miR-103a-2*
CGCTTCACGAATTTGCGTGTCAT

GTCGTATCCAGTGCGTGTCGTGGAGTCGGCAATTGCACTGGATACGACGCTTTGA GTCGTATCCAGTGCGTGTCGTGGAGTCGGCAATTGCACTGGATACGACCCTTCT GTCGTATCCAGTGCGTGTCGTGGAGTCGGCAATTGCACTGGATACGACGATGAA GTCGTATCCAGTGCGTGTCGTGGAGTCGGCAATTGCACTGGATACGACCAAGGC

miR, microRNA.

Table II. Comparison of the clinical characteristics between patients in the RDS and control groups.

\begin{tabular}{lcccccc}
\hline Group & $\begin{array}{c}\text { Number of } \\
\text { patients }\end{array}$ & GA (weeks) & $\begin{array}{c}\text { Gender ratio } \\
\text { (male/female) }\end{array}$ & Weight (g) & 1 min & 5 min \\
\hline RDS & 20 & $31.1 \pm 1.6$ & $15 / 5$ & $1747.5 \pm 434.1$ & $8.10 \pm 0.64$ & $8.80 \pm 0.69$ \\
Control & 29 & $29.5 \pm 7.5$ & $19 / 10$ & $1987.2 \pm 434.4$ & $8.38 \pm 0.90$ & $9.00 \pm 0.84$ \\
t-value & - & 0.092 & $0.751^{\mathrm{a}}$ & 1.899 & 1.190 & 0.873 \\
P-value & - & 0.361 & 0.386 & 0.064 & 0.240 & 0.387 \\
\hline
\end{tabular}

${ }^{\mathrm{a}} \chi^{2}$-value. RDS, respiratory distress syndrome; GA, gestational age.

followed by heat inactivation at $85^{\circ} \mathrm{C}$ for $5 \mathrm{~min}$. For qPCR, $1 \mu \mathrm{l}$ cDNA was added to $24 \mu \mathrm{l}$ aster mix containing $2.5 \mu \mathrm{l}$ dNTP (2.5 mM each), 2.5 $\mu 1$ 10X PCR buffer (Promega Corporation, Madison, WI, USA) and 1 unit Taq polymerase (Promega), final concentration 0.25X Sybergreen1 (Invitrogen Life Technologies) and $2 \mu 1$ reverse and forward primers (Invitrogen Life Technologies). The cDNA was amplified for 35 cycles on an Applied Rotor-Gene 3000 (Corbett Research, Syndey, Australia) PCR system. The primer sequences used are listed in Table I. RT-qPCR for U6 snRNA were performed in each plate as an endogenous control. The quantity of the PCR products were calculated from the threshold cycle $\left(\mathrm{C}_{t}\right)$, and the comparative $\mathrm{C}_{\mathrm{t}}$ method was used. The quantity of each miRNA relative to U6 snRNA was calculated using the equation: 2 - $\left(\mathrm{Ct}^{\text {microRNA }}-\mathrm{Ct}^{\mathrm{U} 6)}\right.$.

Statistical analysis. The data were analyzed using the SPSS 13.0 statistical package (SPSS, Inc., Chicago, MO, USA), and the data from the RT-qPCR was assessed using an independent samples t-test. $\mathrm{P}<0.05$ was considered to indicate a statistically significant difference.

\section{Results}

General features of the clinical data. The present study comprised 20 infants with RDS and 29 infants in total. The mean GAs and weights in the two groups were $31.1 \pm 1.6$ weeks; 1747.5 $\pm 434.1 \mathrm{~g}$ and $29.5 \pm 7.5$ weeks; $1987.2 \pm 434.4 \mathrm{~g}$, respectively. The constituent male/female ratios were $15 / 5$ and 19/10, respectively. The mean 1 min Apgar scores were $8.10 \pm 0.64$ and $8.38 \pm 0.90$, and the mean 5 min Apgar scores were $8.80 \pm 0.69$, and $9.00 \pm 0.84$, respectively. No significant differences were identified in these values between the two groups (Table II).

miRNA expression profile. The sixth generation of the miRCURY ${ }^{\mathrm{TM}}$ LNA array (v.16.0; Exiqon), contains $>1,891$ capture probes, covering all human, mouse and rat miRNAs annotated in miRBase 16.0. As a result, in the three slides analyzed in the present study, 171 differentially expressed miRNAs passed the fold-change filtering, which identifies those with a fold-change $>1.3$ between the two groups, including 116 upregulated and 55 downregulated miRNAs (Table III). From these differentially expressed miRNAs, specific criterion were used to screen specific miRNAs for further investigation. The miRNAs selected were those that passed through Volcano Plot filtering, with a fold-change $\geq 1.3$ and $\mathrm{P} \leq 0.05$. Following screening, two significantly upregulated and seven significantly downregulated miRNAs met the filtering requirements. The detailed fold-changes of these nine miRNAs in microarrays were compared using histograms (Figs. 1 and 2), based on the data in Table III. Hierarchical clustering was then performed to highlight distinguishable miRNA expression profiling between the two groups. In the heat map diagram (Fig. 3), each row represents one of the nine miRNAs and each column represents a slide. The miRNA clustering tree is showed on the left and the slide clustering tree is shown at the top.

$R T-q P C R$. Of the nine miRNAs identified, the four exhibiting the highest fold-changes, regardless of whether upregulated or downregulated, were further selected. These contained two upregulated miRNAs (hsa-miR-513a-3p and hsa-miR-103-2*) and two downregulated miRNAs (hsa-miR-301a and 


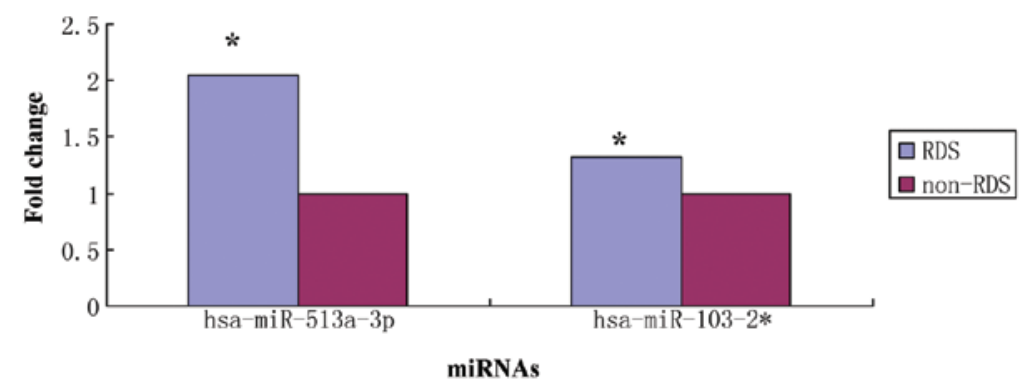

Figure 1. Fold-changes of two upregulated miRNAs between the RDS and non-RDS groups. Higher expression levels were observed in the RDS group $(* \mathrm{P}<0.05)$. miRNA, microRNA; RDS, respiratory distress syndrome.

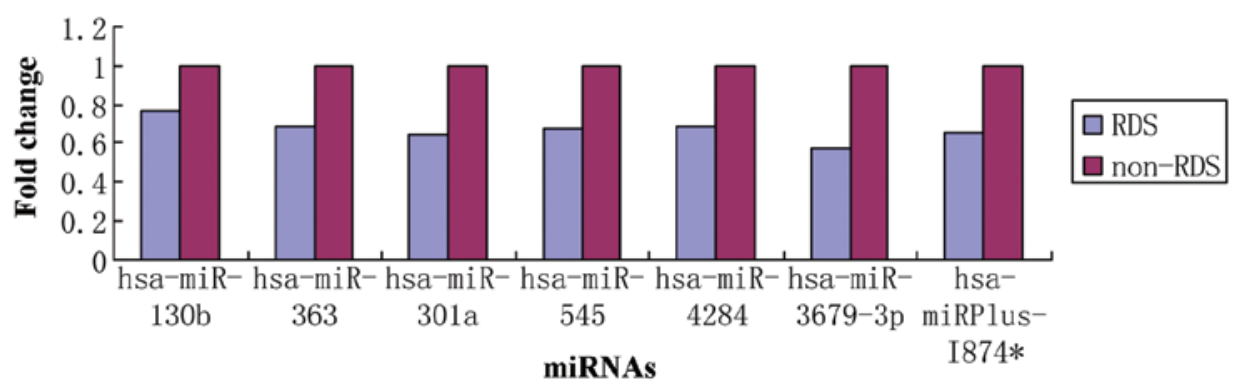

Figure 2. Fold-changes of seven downregulated miRNAs between the RDS and non-RDS groups $(* \mathrm{P}<0.05)$. miRNA, microRNA; RDS, respiratory distress syndrome.

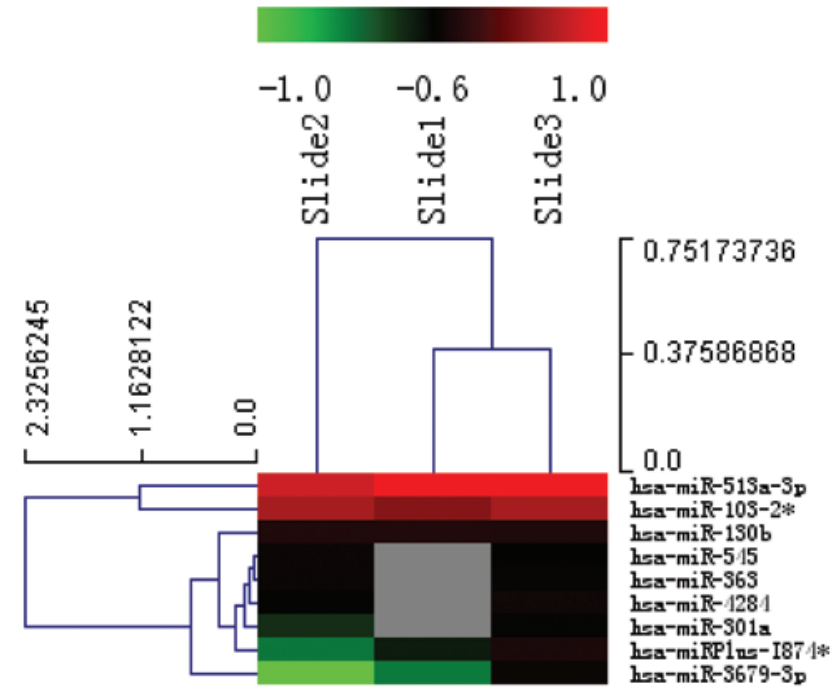

Figure 3. miRNA expression profile. In the heat map diagram, each row represents an miRNA and each column represents a one of the triplicate repeats. The miRNA clustering tree is shown on the left, and the slide clustering tree appears at the top. Red indicates high relative expression, green indicates low relative expression and black represents zero. miRNA, microRNA.

hsa-miR-3679-3p). These four selected miRNAs were subsequently confirmed using RT-qPCR, The relative expression levels of these are shown in Fig. 4.

\section{Discussion}

Clinical advancements in neonatal techniques have improved the prognosis of preterm infants, however, the incidences of BPD and RDS have also gradually increased $(21,22)$. Although

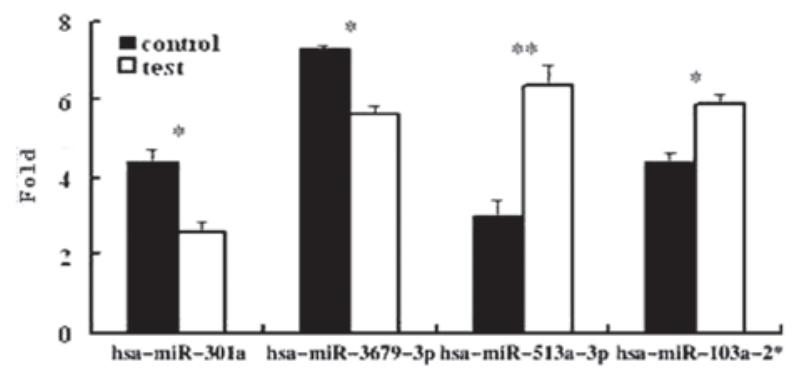

Figure 4. miRNA expression profile of the four selected miRNAs, determined using reverse transcription-quantitative polymerase chain reaction. The test group refers to the respiratory distress syndrome group. $\left({ }^{*} \mathrm{P}<0.05\right.$ and ${ }^{* *} \mathrm{P}<0.01$, compared with the test group). miRNA, microRNA.

there are few statistics from large sample studies in China, in Europe, the incidence of RDS in infants of a GA between 23 and 25 weeks has risen to $91 \%$ per year (23). Currently, it is suggested that RDS is a complex network disease caused by several factors. The disease is characterized by immature lung development and a lack of PS. Previously, the let-7 family and miRNA-17-92 cluster have been demonstrated to be important in lung development (5-8). Ventura et al (24) demonstrated that mice deficient in the miRNA-17-92 cluster exhibit lung hypoplasia defects, characterized by smaller, hypoplastic lungs, ventricular septal defects and abnormal B-cell development. In our preliminary study (25), several novel differentially expressed miRNAs were identified during normal lung development in rats, including miRNA-296 and miRNA-93.

In the present study, nine novel miRNAs were identified, including two upregulated and seven downregulated miRNAs. Among these, hsa-miR-513a-3p, hsa-miR-103-2*, hsa-miR-301a 
Table III. miRNA expression profiles, with fold-changes, of upregulated and downregulated miRNAs between the two groups.

\begin{tabular}{|c|c|}
\hline miRNA & Fold-chang \\
\hline \multicolumn{2}{|l|}{ Upregulated } \\
\hline hsa-miR-3171 & 102.393 \\
\hline hsa-miR-938 & 33.867 \\
\hline hsa-miR-937 & 23.561 \\
\hline hsa-miR-513b & 19.954 \\
\hline hsa-miR-3681 & 9.188 \\
\hline hsa-miRPlus-K1303* & 8.900 \\
\hline hsa-miR-1275 & 8.203 \\
\hline hsa-miR-330-3p & 5.242 \\
\hline hsa-miR-4275 & 5.239 \\
\hline hsa-miRPlus-A1072 & 5.050 \\
\hline hsa-miR-138-2* & 4.169 \\
\hline hsa-miR-4288 & 3.941 \\
\hline hsa-miR-203 & 3.522 \\
\hline hsa-miR-483-3p & 3.487 \\
\hline hsa-miR-589 & 3.104 \\
\hline hsa-miR-3907 & 2.989 \\
\hline hsa-miR-132 & 2.988 \\
\hline hsa-miR-23a & 2.942 \\
\hline hsa-miR-183 & 2.733 \\
\hline hsa-miR-3178 & 2.690 \\
\hline hsa-miR-214 & 2.658 \\
\hline hsa-miR-491-3p & 2.584 \\
\hline hsa-miRPlus-I382* & 2.569 \\
\hline hsa-miR-769-3p & 2.562 \\
\hline hsa-miR-640 & 2.423 \\
\hline hsa-miR-520d-5p & 2.371 \\
\hline hsa-miR-720 & 2.273 \\
\hline hsa-miR-487b & 2.268 \\
\hline hsa-miR-3915 & 2.219 \\
\hline hsa-miR-574-3p & 2.196 \\
\hline hsa-miR-1260b & 2.185 \\
\hline hsa-miR-1246 & 2.166 \\
\hline hsa-miR-519e & 2.139 \\
\hline hsa-miR-138-1* & 2.105 \\
\hline hsa-miR-154 & 2.062 \\
\hline hsa-miR-513a-3p & 2.046 \\
\hline hsa-miR-513a-5p & 2.011 \\
\hline hsa-miR-519e* & 1.985 \\
\hline hsa-miR-597 & 1.964 \\
\hline hsa-miR-3685 & 1.953 \\
\hline hsa-miR-4324 & 1.922 \\
\hline hsa-miR-23c & 1.907 \\
\hline hsa-miR-4291 & 1.868 \\
\hline hsa-miR-1260 & 1.839 \\
\hline hsa-miR-642b & 1.838 \\
\hline hsa-miR-629* & 1.832 \\
\hline hsa-miR-1290 & 1.816 \\
\hline hsa-miR-16 & 1.810 \\
\hline hsa-miR-99b* & 1.808 \\
\hline hsa-miR-2115* & 1.807 \\
\hline hsa-miR-559 & 1.717 \\
\hline hsa-miR-508-5p & 1.712 \\
\hline hsa-miR-631 & 1.710 \\
\hline
\end{tabular}

Table III. Continued.$$
\text { miRNA }
$$

Fold-change

hsa-miR-221 1.709

hsa-miR-424 1.703

hsa-miR-891a $\quad 1.696$

hsa-miR-518a-5p/hsa-miR-527 1.693

hsa-miR-933 1.656

hsa-let-7i* 1.641

hsa-miR-675 1.641

hsa-miR-224* $\quad 1.609$

hsa-miR-211 1.585

hsa-miRPlus-A1015

hsa-miR-711 $\quad 1.574$

hsa-miR-3646 $\quad 1.538$

hsa-miR-1284 $\quad 1.521$

hsa-miR-25* $\quad 1.519$

hsa-miR-345 $\quad 1.517$

hsa-let-7d* $\quad 1.515$

hsa-miR-373* $\quad 1.512$

hsa-miR-665 1.506

hsa-miR-492 1.505

hsa-miR-29b 1.496

hsa-miR-144 1.482

hsa-miR-452 1.479

hsa-miR-425* $\quad 1.470$

hsa-miR-7-2* 1.464

hsa-miR-3615 1.462

hsa-miR-502-5p 1.457

hsa-miR-196a* $\quad 1.443$

hsa-miR-638 1.443

hsa-miR-3161 1.440

hsa-miR-1273e 1.439

hsa-miR-501-5p $\quad 1.438$

hsa-miR-525-5p $\quad 1.432$

hsa-miR-1280 1.430

hsa-let-7e $\quad 1.417$

hsa-miR-155 1.413

hsa-miR-1274b $\quad 1.405$

hsa-miR-625 1.404

hsa-miR-3935 1.402

hsa-miR-92a $\quad 1.402$

hsa-miR-101 1.401

hsa-miR-302c* $\quad 1.400$

hsa-miR-451 1.396

hsa-miR-181a $\quad 1.396$

hsa-miR-487a $\quad 1.394$

hsa-miR-600 1.393

hsa-miR-1914 1.387

hsa-miR-125a-5p $\quad 1.384$

hsa-miR-146a $\quad 1.374$

hsa-miR-3686 1.364

hsa-miR-16-1* $\quad 1.362$

hsa-miR-223 1.352

hsa-miR-187* 1.340 
Table III. Continued.

\begin{tabular}{|c|c|}
\hline miRNA & Fold-chang \\
\hline hsa-let-7a-2* & 1.342 \\
\hline hsa-miR-4325 & 1.337 \\
\hline hsa-miRPlus-J1011 & 1.332 \\
\hline hsa-miR-3653 & 1.329 \\
\hline hsa-miR-1273c & 1.328 \\
\hline hsa-miR-3196 & 1.327 \\
\hline hsa-miR-103-2* & 1.319 \\
\hline hsa-miR- $625^{*}$ & 1.315 \\
\hline hsa-miR-409-3p & 1.314 \\
\hline hsa-miR-3202 & 1.310 \\
\hline hsa-miR-634 & 1.309 \\
\hline \multicolumn{2}{|l|}{ Downregulated } \\
\hline hsa-miR-885-5p & 0.459 \\
\hline hsa-miRPlus-A1073 & 0.483 \\
\hline hsa-miR-1281 & 0.487 \\
\hline hsa-miRPlus-A1087 & 0.537 \\
\hline hsa-miR-3940 & 0.556 \\
\hline hsa-miR-337-5p & 0.570 \\
\hline hsa-miR-136* & 0.571 \\
\hline hsa-miR-3679-3p & 0.575 \\
\hline hsa-miR-718 & 0.578 \\
\hline hsa-miR-376b & 0.578 \\
\hline hsa-miR-940 & 0.590 \\
\hline hsa-miR-432 & 0.599 \\
\hline hsa-miR-379 & 0.600 \\
\hline hsa-miR-376a* & 0.624 \\
\hline hsa-miR-3201 & 0.641 \\
\hline hsa-miR-325 & 0.645 \\
\hline hsa-miR-3620 & 0.647 \\
\hline hsa-miR-301a & 0.648 \\
\hline hsa-miR-2113 & 0.653 \\
\hline hsa-miRPlus-I874* & 0.657 \\
\hline hsa-miR-301b & 0.658 \\
\hline hsa-miR-3663-5p & 0.666 \\
\hline hsa-miR-519d & 0.672 \\
\hline hsa-miR-493 & 0.673 \\
\hline hsa-miR-620 & 0.674 \\
\hline hsa-miR-545 & 0.678 \\
\hline hsa-miR-32 & 0.682 \\
\hline hsa-miR-4284 & 0.685 \\
\hline hsa-miR-363 & 0.687 \\
\hline hsa-miR-4255 & 0.691 \\
\hline hsa-miR-548e & 0.695 \\
\hline hsa-miRPlus-I107* & 0.695 \\
\hline hsa-miR-24-2* & 0.700 \\
\hline hsa-let-7b* & 0.704 \\
\hline hsa-miR-552 & 0.708 \\
\hline hsa-miR-7 & 0.717 \\
\hline hsa-miR-431 & 0.718 \\
\hline hsa-miR-361-3p & 0.719 \\
\hline hsa-miR-190 & 0.724 \\
\hline hsa-miR-126 & 0.728 \\
\hline
\end{tabular}

Table III. Continued.

\begin{tabular}{lc}
\hline miRNA & Fold-change \\
\hline hsa-miR-20b & 0.729 \\
hsa-miR-299-3p & 0.731 \\
hsa-miR-744 & 0.732 \\
hsa-miR-1470 & 0.734 \\
hsa-miR-146b-3p & 0.743 \\
hsa-miR-374c & 0.744 \\
hsa-miRPlus-B1114 & 0.745 \\
hsa-miR-323-3p & 0.746 \\
hsa-miR-381 & 0.750 \\
hsa-miR-140-5p & 0.754 \\
hsa-miR-542-3p & 0.757 \\
hsa-miR-377 & 0.764 \\
hsa-miRPlus-A1086 & 0.764 \\
hsa-miR-505* & 0.768 \\
hsa-miR-130b & 0.769 \\
\hline
\end{tabular}

Differentially expressed miRNAs passed fold-change filtering (fold-change $>1.3$ between the two groups). miRNA/miR, microRNA.

and hsa-miR-3679-3p were further analyzed, as they exhibited higher fold-changes in the microarray. Few previous studies have investigated miR-513a-3p. A study by Zhang et al demonstrated that miR-513a-3p can sensitize human lung adenocarcinoma cells to cisplatin by targeting glutathione S-transferase P1, which has been reported to contribute to cisplatin resistance in a number of studies (25-28). miR-103-2*, which has also received less investigation, is generally induced in response to hypoxia (29) and is also involved in pyruvate and lipid metabolism (30). Ellis et al reported that miR-103 changes significantly in patients with heart failure (HF), compared with patients with non-HF dyspnoea and healthy controls (31). Further clarification of the development of RDS may be beneficial.

The present study demonstrated for the first time, to the best of our knowledge, that miR-3679-3p is significantly expressed during the pathological process of RDS. Hsa-miR-301a is a newly identified miRNA, of which the majority of associated studies have focussed on cancer. Lu et al demonstrated that miR-301a downregulates nuclear factor- $\kappa \mathrm{B}$ repressing factor (NKRF) and elevates the activation of $N F-\kappa B$ in cancer cells (32). This is considered to interact with specific negative regulatory elements to mediate the transcriptional activity of $\mathrm{NF}-\kappa \mathrm{B}$, which regulates the expression of three $\mathrm{NF}-\kappa \mathrm{B}$-responsive genes, interleukin (IL)-8, interferon-b, and nitric oxide synthase $2 \mathrm{~A}$. IL- 8 is a cytokine with high levels of expression in the lung tissues of patients of model animals with RDS $(33,34)$.

In addition to the four novel miRNAs evaluated, the five remaining downregulated miRNAs identified in the present study may also be involved in RDS due to their significant fold-changes between the RDS and control groups. Takahashi et al reported that miRNA-363 is overexpressed in CD4 $\left(^{+}\right)$and CD8 $\left(^{+}\right) \mathrm{CB}$ cells in human cord blood and adult 
peripheral blood cells upon proinflammatory stimulation (35), which suggested its immunomodulatory role in inflammatory diseases, including RDS. As for miRNA-130b, miRNA-545, miRNA-4284 and miRNA-I874*, previous reports have demonstrated their association with cancer, rather than lung diseases and are, therefore, not discussed further.

In conclusion, the present study identified several differentially expressed miRNAs between the plasmaa of RDS and control groups for the first time, to the best of our knowledge. These results support the hypothesis that, to some degree, these novel miRNAs may be involved in the pathogenesis of RDS, and may assist in providing meaningful biomarkers for the diagnosis of RDS.

\section{Acknowledgements}

This study was supported by a grandt from the Project Foundation of Jiangsu Province Health Department (no. H200642)

\section{References}

1. Sweet D, Carnielli V, Greisen G, et al: European consensus guidelines on the management of neonatal respiratory distress syndrome in preterm infants - 2013 update. Neonatology 103: 353-368, 2013.

2. Soll RF: Early versus delayed selective surfactant treatment for neonatal respiratory distress syndrome. Neonatology 104 124-126, 2013.

3. Chang LW and Li WB: Fetal and neonatal lung development J Appl Clin Pediatrics 26: 1065-1067, 2011.

4. Morrisey EE: Wnt signaling and pulmonary fibrosis. Am J Pathol 162: 1393-1397, 2003.

5. Chen XM, Splinter PL, O'Hara SP, et al: A cellular micro-RNA, let-7i, regulates toll-like receptor 4 expression and contributes to cholangiocyte immune responses against cryptosporidium parvum infection. J Biol Chem 282: 28929-28938, 2007.

6. Johnson CD, Esquela-Kerscher A, Stefani G, et al: The let-7 microRNA represses cell prolif-eration pathways in human cells. Cancer Res 67: 7713-7722, 2007.

7. Lu Y, Thomson JM, Wong HY, et al: Transgenic overexpression of the microRNA miR-17-92 cluster promotes proliferation and inhibits differentiation of lung epithe-lial progenitor cells. Dev Biol 310: 442-453, 2007.

8. Ramasamy SK, Mailleux AA, Gupte VV, et al: Fgf10 dosage is critical for the amplification of epithelial cell progenitors and for the formation of multiple mesenchymal lineages during lung development. Dev Biol 307: 237-247, 2007.

9. Sayed D and Abdellatif M: MicroRNAs in development and disease. Physiol Rev 91: 827-887, 2011.

10. Lim LP, Lau NC, Garrett-Engele P, et al: Microarray analysis shows that some microRNAs downregulate large numbers of target mRNAs. Nature 433: 769-773, 2005.

11. Ambros V: The functions of animal microRNAs. Nature 431: $350-355,2004$

12. Taylor DD and Gercel-Taylor C: MicroRNA signatures of tumor derived exosomes as diagnostic biomarkers of ovarian cancer. Gynecol Oncol 110: 13-21, 2008.

13. Hiroki E, Akahira J, Suzuki F, et al: Changes in microRNA expression levels correlate with clinicopathological features and prognoses in endometrial serous adenocarci nomas. Cancer Sci 101: 241-249, 2010.
14. Grosshans H and Filipowicz W: Molecular biology: the expanding world of small RNAs. Nature 451: 414-416, 2008.

15. Mandel EE and Paris Da: The routine use of the serum flocculation reaction with Hayem's solution. J Lab Clin Med 33: 1629, 1948.

16. Chen X, Ba Y, Ma L, et al: Characterization of microRNAs in serum: a novel class of biomarkers for diagnosis of cancer and other diseases. Cell Res 18: 997-1006, 2008.

17. El-hefnawy T, Raja S, Kelly L, et al: Characterization of amplifiable, Circulating RNA in plasma and its potential as a tool for cancer diagnostics. Clin Chem 50: 564-573, 2004.

18. György B, Szabó TG, Pásztói M, Pál Z, Misják P, Aradi B, László V, Pállinger E, Pap E, Kittel A, Nagy G, Falus A and Buzás EI. Membrane vesicles, current state-of-the-art: emerging role of extracellular vesicles. Cell Mol Life Sci 68: 2667-2688, 2011.

19. Ai J, Zhang R, Li Y, et al: Circulating microRNA-1 as a potential novel biomarker for acute myocardial infarction. Biochem Biophys Res Commun 391: 73-77, 2010.

20. Yuan L and Chen C: Prevention and treatment of neonatal respiratory distress syndrome in Europe guide. Chin J Pediatr 49: 27-33, 2011.

21. World Medical Association. World Medical Association Declaration of Helsinki: Ethical principles for medical research involving human subjects. JAMA 310: 2191-2194, 2013.

22. Jobe AH: The new bronchopulmonary dysplasia. Curr Opin Pediatr 23: 167-172, 2011

23. Ruegger C, Hegglin M, Adams M, et al: Population based trends in mortality, morbidity and treatment for very preterm-and very low birth weight infants over 12 years. BMC Pediatr 12: 17, 2012.

24. Ventura A, Young AG, Winslow MM, et al: Targeted deletion reveals essential and overlapping functions of the miR-17 through 92 family of miRNA clusters. Cell 132: 875-886, 2008.

25. Zhang X, Zhu J, Xing R, et al: miR-513a-3p sensitizes human lung adenocarcinoma cells to chemotherapy by targeting GSTP1. Lung Cancer 77: 488-494, 2012.

26. Yang Y, Kai G, Pu XD, et al: Expression profile of microRNAs in fetal lung development of Sprague-Dawley rats. Int J Mol Med 29: 393-402, 2012.

27. Kulshreshtha R, Ferracin M, Wojcik SE, et al: A microRNA signature of hypoxia. Mol Cell Biol 27: 1859-1867, 2007.

28. Huang G, Mills L and Worth LL. Expression of human glutathione S-transferase P1 mediates the chemosensitivity of osteosarcoma cells. Mol Cancer Ther 6: 1610-1619, 2007.

29. Ishii T, Fujishiro M, Masuda M, et al: A methylated oligonucleotide induced methylation of GSTP1 promoter and suppressed its expression in A549 lung adenocarcinoma cells. Cancer Lett 212: 211-223, 2004

30. Wilfred BR, Wang WX and Nelson PT: Energizing miRNA research: a review of the role of miRNAs in lipid metabolism, with a prediction that miR-103/107 regulates human metabolic pathways. Mol Genet Metab 91: 209-217, 2007.

31. Ellis KL, Cameron VA, Troughton RW, Frampton CM, et al: Circulating microRNAs as candidate markers to distinguish heart failure in breathless patients. Eur J Heart Fail 15: 1138-1147, 2013.

32. Lu Z, Li Y, Takwi A, et al: miR-301a as an NF- kB activator in pancreatic cancer cells. EMBO J 30: 57-67, 2011.

33. Kotecha S: cytokine in chronic lung disease of prematurity. Eur J Pediatr 155 suppl 2: 14-17, 1996.

34. Munshi UK, Niu JO, Siddiq MM, et al: Elevation of interleukin- 8 and interleukin- 6 precedes the influx of neutrophils in tracheal aspirates from preterm infants who develop bronchopulmonary dysplasia. Pediatr Pulmonol 24: 331-336, 1997.

35. Takahashi N, Nakaoka $\mathrm{T}$ and Yamashita N: Profiling of immune-related microRNA expression in human cord blood and adult peripheral blood cells upon proinflammatory stimulation. Eur J Haematol 88: 31-38, 2012. 